\title{
Editorial
}

\section{Monitored anaesthesia care (MAC)}

Monitored anaesthesia care (MAC) refers to a clinical service where in an anaesthesiologist provides analgesia and sedation for a diagnostic or therapeutic procedure, and the patient is able to protect his airway for the majority of the procedure. ${ }^{1}$ It involves the administration of medication that can potentially lead to loss of consciousness and normal protective reflexes. There is possibility of a deeper plane of sedation compared to 'sedation/analgesia' which is provided by a non-anaesthesiologist. ${ }^{2}$ Therefore, the ASA recommends that the standards of care be same as for general or regional anaesthesia, with regard to pre-operative evaluation, intra-operative monitoring of the cardio-respiratory system, the physical presence of an anaesthesiologist at all time, and the administration of oxygen and other medications to keep the patient safe and comfortable. ${ }^{1} \mathrm{MAC}$ should invoke less physiological disturbance and allow a more rapid recovery compared to a general anaesthetic. Therefore, it is not surprising to note that it is the technique of choice in up to $30 \%$ of the surgical procedures.

According to the American Society of Anesthesiologists (ASA), a monitored anesthesia care (MAC) is a planned procedure during which the patient undergoes local anesthesia together with sedation and analgesia. Actually MAC is the first choice in 10-30\% of all the surgical procedures. The 3 fundamental elements and purposes of a conscious sedation during a MAC are: a safe sedation, the control of the patient anxiety and the pain control. The patients undergoing conscious sedation are able to answer to orders appropriately and to protect airways. Last but not least, another purpose of any MAC is to get the patient appropriately satisfied, allowing him to get his discharge as faster as possible. There are many surgical procedures which can be performed using a MAC. The patient consciousness evaluation is of extreme importance during the surgical procedure performed with MAC: to this purpose the clinical and electroencephalographic evaluations such as Bispectral Index are very useful. MAC can be obtained with the association of fast half-life drugs or drugs getting a clinical effect which can vary according to the surgical requirements, using an infusion regiment. Apart from the pharmacological choice, this procedure can be performed with patient controlled sedation techniques or with continued intravenous infusion or with target controlled infusion.

Monitored Anesthesia Care (MAC) is a type of sedation that is administered through an IV to make a patient sleepy and calm during a procedure. The patients are typically awake, but groggy, and are able to follow instructions as needed.

The level of sedation provided with this type of anesthesia can range from light, where the patient just feels very relaxed, to heavy, where the patient is unaware of what is happening and only rouses to significant stimulation.

Because the level of sedation varies, the process is monitored, with a anesthesia professional present to continuously monitor the patient's vital signs and maintain or adjust the level of sedation as needed. This type of sedation is frequently used with minor surgical procedures and dental procedures and can be combined with local or regional anesthesia.

Depending on the medications used as the doses given, the patient may or may not remember the procedure. The American College of Gastroenterology (ACG) released a Position Statement (Vargo, 2009) which recommends that "the use of anesthesiologist-administered sedation for healthy, low-risk patients undergoing routine GI endoscopy results in higher costs with no proven benefit with respect to patient safety or procedural efficacy."

Paspatis and colleagues (2011) reported on 520 individuals undergoing colonoscopy for the detection of polyps and were randomized to either deep sedation $(\mathrm{n}=258)$ or moderate sedation $(\mathrm{n}=262)$ with the hypothesis that deep sedation may increase the rate of polyp detection compared to moderate sedation which would enhance the 
quality of the colonoscopy. The degree of sedation was assessed by a research nurse using the modified assessment of alertness/sedation (MOAA/ S) scale. Each participant's satisfaction with the sedation was assessed 2 hours after the procedure in the recovery area. The endoscopist's satisfaction concerning the sedation during the procedure was also assessed immediately following the procedure. There was no difference between the two groups in regards to the overall rate of polyps detected. There were no differences in levels of participant satisfaction between the two groups. However, the endoscopist's satisfaction rating was greater in the deep sedation group compared to the moderate sedation group.

Local anesthesia with sedation offers anesthesia personnel and the surgeon great flexibility in tailoring the degree of anesthesia to the needs of the patient. Procedures that once required patients to stay overnight in the hospital are now performed safely in office and outpatient surgical suites. $\underline{5}$ The introduction of new anesthetic applications enables patients to undergo lengthy and complex procedures as outpatients and then promptly and safely be discharged home. $\frac{6}{-T h e}$ choice and route of anesthesia administration is paramount to the patient's overall surgical experience. If, upon discharge, the patient is alert, has minimal pain, and has no nausea or vomiting, then the surgical experience was a positive one. ${ }^{-}$

The three essential components of MAC are - safe sedation, anxiolysis and analgesia. ${ }^{3}$ However, patient comfort, cardio-respiratory stability, good operating conditions and minimal side effects are equally important.

Monitored anesthesia care (MAC) refers to the anesthesia personnel present during a procedure and does not implicitly indicate the level of anesthesia needed. Monitored anesthesia care is a specific anesthesia service for a diagnostic or therapeutic procedure. Indications for monitored anesthesia care include the nature of the procedure, the patient's clinical condition and/or the potential need to convert to a general or regional anesthetic. Monitored anesthesia care includes all aspects of anesthesia care-a preprocedure visit, intra-procedure care and postprocedure anesthesia management. During monitored anesthesia care, the anesthesiologist provides or medically directs a number of specific services, including but not limited to: Diagnosis and treatment of clinical problems that occur during the procedure; support of vital functions; administration of sedatives, analgesics, hypnotics, anesthetic agents or other medications as necessary for patient safety; psychological support and physical comfort; and provision of other medical services as needed to complete the procedure safely.

MAC may include varying levels of sedation, analgesia, and anxiolysis as necessary. The provider of MAC must be prepared and qualified to convert to general anesthesia when necessary. If the patient loses consciousness and the ability to respond purposefully, the anesthesia care is a general anesthetic, irrespective of whether airway instrumentation is required.

Use of monitored anesthesia care may be considered medically necessary for gastrointestinal endoscopy, bronchoscopy, and interventional pain procedures, when there is documentation by the procedural list and anesthesiologist that specific risk factors or significant medical conditions are present. Those risk factors or significant medical conditions include any of the following:

Increased risk for complications due to severe comorbidity (ASA P3* or greater); morbid obesity (BMI [body mass index] > 40); documented sleep apnea; inability to follow simple commands (cognitive dysfunction, intoxication, or psychological impairment); spasticity or movement disorder complicating procedure; history or anticipated intolerance to standard sedatives, such as: chronic opioid use, chronic benzodiazepine use; patients with active medical problems related to drug or alcohol abuse; patients younger than 18 years or 70 years or older; patients who are pregnant; patients with increased risk for airway obstruction due to anatomic variation, such as: history of stridor, dysmorphic facial features, oral abnormalities (e.g., macroglossia),neck abnormalities (e.g., neck mass), jaw abnormalities (e.g., micrognathia); Acutely agitated, uncooperative patients; prolonged or therapeutic gastrointestinal endoscopy procedures requiring deep sedation.

Monitored anesthesia care can be provided by qualified anesthesia personnel with training and experience in: patient assessment; continuous evaluation and monitoring of patient physiological functions; diagnosis and treatment (both pharmacological and non-pharmacological) of any and all deviations in physiological function. 
Examples of prolonged endoscopy procedures that may require deep sedation include adhesions postabdominal surgery, endoscopic retrograde cholangiopancreatography, stent placement in the upper GI tract, and complex therapeutic procedures such as plication of the cardioesophageal junction.

The Mallampati score is considered a predictor of difficult tracheal intubation and is routinely used in preoperative anesthesia evaluation. ${ }^{4}$ The score is obtained by having the patient extend the neck, open the mouth, and extend the tongue while in a seated position. Patients are scored from Class 1-4 as follows: Class I - the tonsils, uvula and soft palate are fully visible; Class 2 - the hard and soft palate, uvula and upper portion of the tonsils are visible; Class 3 - the hard and soft palate and the uvula base are visible; Class 4 - only the hard palate is visible.

Patients with Class 3 or 4 Mallampati scores are considered to be at higher risk of intubation difficulty. While the Mallampati score does not determine a need for monitored anesthesia care, it may be considered in determining risk for airway obstruction. Other tests to predict difficult tracheal intubation include the upper lip bite test, the intubation difficulty scale, and the CormackLehane grading system.

\section{Dr. M. Manzoorul Hoq Laskar}

Senior Consultant, Department of Anaesthesia, Analgesia and Intensive Care

Bangabandhu Sheikh Mujib Medical University

E-mail : manzoorulhoq@ymail.com

Face book : Manzoor.Laskar@facebook.com

\section{References}

1. Novak LC. ASA position on Monitored Anaesthesia Care. ASA newsletter 62; Dec 1998

2. Hillier SC, Mazurek MS. Monitored anesthesia care, In Barash PG, Cullen BF, Stoelting RK (eds) Clinical Anesthesia, Lippincott Williams \& Wilkin 2006; 1246-61

3. Ghisi D, Fanelli M, Tosi M, Nuzzi M, Panelli G. Monitored anaesthesia care. Minerva Anesthesiologica 2005; 71: 533-38

4. Mallampati SR, Gatt SP, Gugino LD et al. A clinical sign to predict difficult tracheal intubation: a prospective study. Can Anaesth Soc J 1985; 32(4):429-34

5. Bitar G, Mullis W, Jacobs W, et al. Safety and efficacy of office-based surgery with monitored anesthesia care/sedation in 4778 consecutive plastic surgery procedures. Plast Reconstr Surg. Jan 2003;111(1):150-6; discussion 157-8

6. Cinnella G, Meola S, Portincasa A, et al. Sedation analgesia during office-based plastic surgery procedures: comparison of two opioid regimens. Plast Reconstr Surg. Jun 2007; 119(7): 2263-70

7. Stoelting RK. Intrathecal morphine-an underused combination for postoperative pain management. Anesth Analg. Jun 1989; 68(6): 707-9 